\title{
Xanthogranulomatous cholecystitis associated with carcinoma of the gallbladder
}

\author{
E.W. Benbow \\ Department of Pathology, University of Manchester, Stopford Building, Oxford Road, Manchester M13 9PT, UK.
}

\begin{abstract}
Summary: When the histological material from 35 examples of carcinoma of the gallbladder was reviewed, three specimens were found to show xanthogranulomatous cholecystitis as well as the tumour, suggesting that these two lesions are associated with each other. This is important because xanthogranulomatous cholecystitis can mimic carcinoma, and there is potential for diagnostic confusion.
\end{abstract}

\section{Introduction}

Xanthogranulomatous cholecystitis (XGC) is the name generally used to describe the lesion which results when lipids from the bile in the lumen of the gallbladder enter the wall of the organ and induce a granulomatous inflammation. ${ }^{1}$ This lesion may simulate carcinoma clinically and on macroscopic examination of a specimen; even its histological features may suggest invasive tumour to the unwary. The occasional co-existence of xanthogranulomatous cholecystitis and carcinoma in the gallbladder ${ }^{2,3}$ adds a further dimension to the potential for diagnostic confusion. I have reviewed the histological material from 35 cases of gallbladder carcinoma to determine the frequency of coexistent xanthogranulomatous cholecystitis, and to determine whether this reduced the accuracy of the original histological reports.

\section{Materials}

Biopsy diagnoses recorded at Manchester Royal Infirmary have been indexed for the last 37 years, and include 43 cases of gallbladder carcinoma. Eight of these diagnoses were made on tiny scraps of material removed at 'open-and-shut' laparotomies, and these cases have not been considered further. In the remaining 35 cases, cholecystectomy had been performed; carcinoma was confirmed in each case, and that part of the gallbladder wall not infiltrated by the tumour was well represented in the histological material. All the available sections from each of these cases were examined, and xanthogranulomatous cholecystitis was found in three of them.

Correspondence: E.W. Benbow, B.Sc., M.B., Ch.B., M.R.C.Path.

Accepted: 30 January 1989

\section{Clinical findings}

Case 1: An 87 year old woman was admitted to hospital with a history of right-sided abdominal pain. This pain had been present for 3 days, and radiated through to the back. At laparotomy, there was a mass in the right side of the abdomen which incorporated the gallbladder, part of the colon and the omentum, together with an abscess cavity. Cholecystectomy was performed, and a gallbladder measuring $7 \times 3.5 \mathrm{~cm}_{6}$ was received in the laboratory. The mucosa was noted? to be brown and granular, and a papillary lesion was found near the resection line. The wall was thickened, and a firm grey-brown area was seen within it. The reporting pathologist made the diagnosis of adenocarcinoma with an 'adenomyoma' at the neck. XGC was not mentioned. The patient died 101 weeks after surgery.

Case 2: A 72 year old woman presented to hospital following three acute episodes of right hypochondrial pain. A mass was palpated in the right hypochondrium which, on ultrasonography, was thought to be a mucocele. At laparotomy, a gallbladder tumour was noted, and a large tumour mass was found in the right lobe of the liver. Cholecystectomy and peritoneal biopsy were performed. When received in the laboratory, the gallbladder was noted to measure $5.1 \times 3 \mathrm{~cm}$; its serosa was roughened, and the wall thickened to a depth of $0.8 \mathrm{~cm}$. The mucosa was replaced by a 'roughened shaggy yellow lining' which, on microscopy, was noted to be formed of granulation tissue. 'Patchy infiltration by moderately differentiated adenocarcinoma' was also recorded. The peritoneal biopsy showed non-specific inflammation. XGC was not mentioned. She was discharged from hospital, and seen in the out-patient

C The Fellowship of Postgraduate Medicine, 1989 
clinic 24 weeks later. She did not attend for subsequent appointments.

Case 3: An 82 year old man presented with nonspecific symptoms, and a mass was found in the right hypochondrium: ultrasonographic examination showed this to be a thick-walled gallbladder with a nodular lesion at its neck (Figure 1). Cholecystectomy was carried out, but his condition remained poor, and he died 24 weeks later without any clinical evidence of metastatic carcinoma. There was no autopsy. The details of this case, particularly the pathological findings, have already been reported. ${ }^{2}$

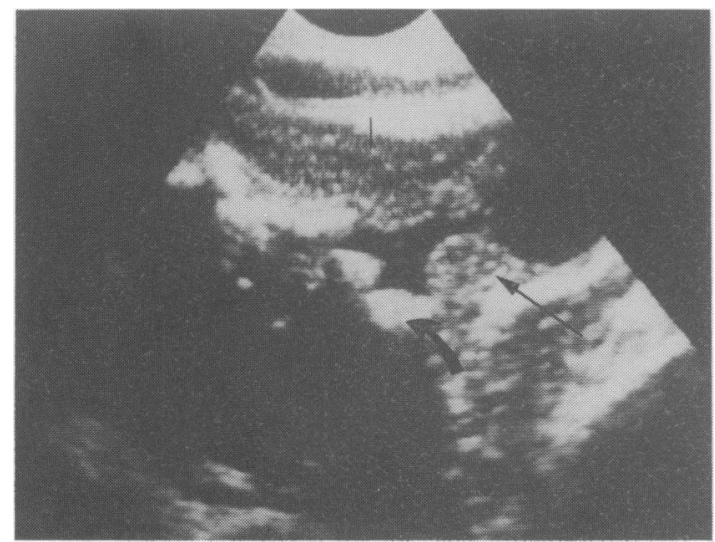

Figure 1 This ultrasonograph of the gallbladder in Case 3 shows thickening of the wall (short arrow), subsequently demonstrated to be largely the result of xanthogranulomatous inflammation. A nodular area at the neck (long arrow) proved to be carcinoma; some gallstones are also present (curved arrow).

\section{Microscopic findings}

Histological review of all three cases showed well differentiated adenocarcinoma which had extensively replaced the mucosa. In case 1 , it had infiltrated through the full thickness of the wall to the serosa, whereas in the other two cases, invasion was limited to the inner half of the wall. In each case, there was at least one point where tumour was close to a focus of XGC. The extent of carcinoma had been accurately assessed by the original pathologist in each case, even though the presence of XGC was not noted in the first two cases.

The overall features of XGC were similar in all three cases, consisting of extensive ill-defined aggregates of large round macrophages with pale-staining granular cytoplasm (Figure 2); more elongated forms which have some resemblance to a spindle-celled tumour were also seen (Figure 3). There were also some

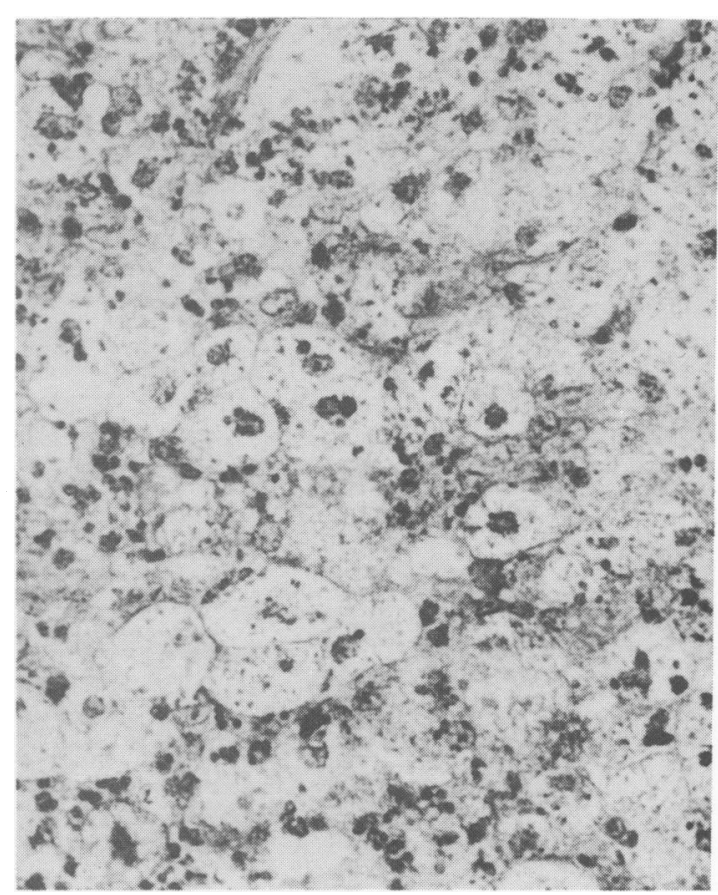

Figure 2 The most prominent component of each focus of XGC consisted of sheets of faintly granular palestaining macrophages (Case 3). Haematoxylin and eosin $\times 392$.

multinucleate foreign-body type giant cells with, in case 1, small foci of Touton-type giant cells (Figure 4).

The location of XGC was different in each example. In case 1 , it formed a rounded area measuring $0.9 \times 0.7 \mathrm{~cm}$ immediately below the mucosa where it had undergone malignant transformation, whereas in case 2 , it was a plaque measuring $1.2 \times 0.35 \mathrm{~cm}$ forming the base of an ulcer immediately adjacent to carcinoma. In case 3 , the XGC was largely limited to the outer half of the wall, where it invested the entirety of the organ.

\section{Discussion}

There is no information in the literature about the overall incidence of xanthogranulomatous cholecystitis, though there are several small series of selected cases $^{3-7}$ and some single case reports ${ }^{2,8,9}$ in the pathological literature, together with two retrospective studies of gallbladders removed for symptomatic gallbladder disease. ${ }^{1,10}$

Roberts and Parsons ${ }^{1}$ studied 723 surgically resected gallbladders in Sheffield in a unit closely similar to the one served by this Department. They found 13 examples of xanthogranulomatous cholecy- 


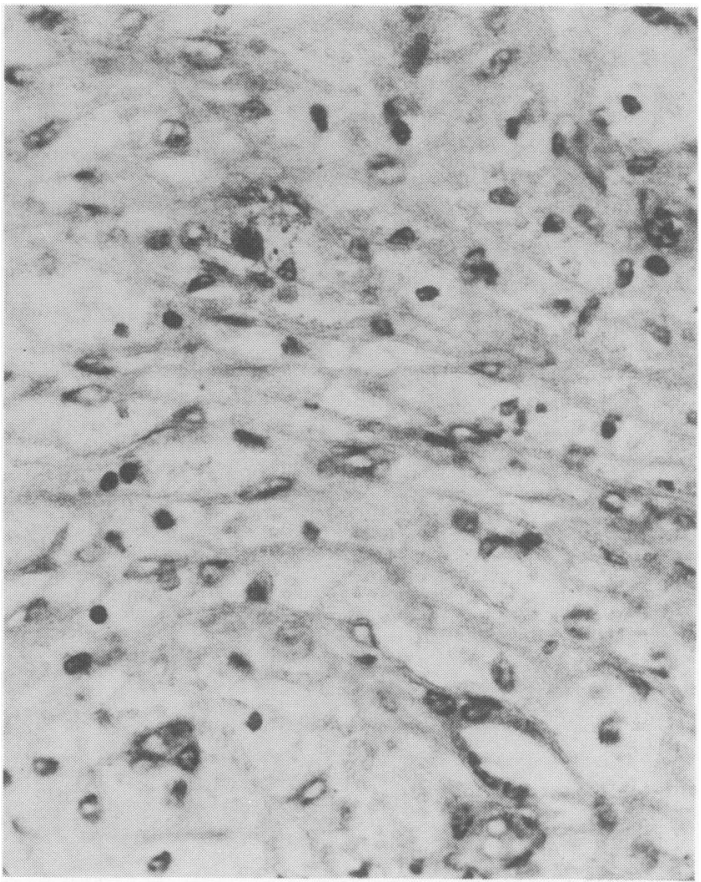

Figure 3 Pale-staining spindle-shaped cells form a minor but consistent component of XGC. Scattered lymphocytes are also present (Case 2). Haematoxylin and eosin $\times 392$.

stitis, so that the incidence of XGC in these neoplasmfree gallbladders was $1.8 \%$, contrasting with the incidence of $8.6 \%$ in carcinomatous gallbladders found in this study. More recently, the same authors ${ }^{11}$ have studied 29 examples of XGC, and found coexistent carcinoma in 3 of them. Although these small numbers do not allow definite conclusions to be drawn, they support the suggestion that XGC is more strongly associated with gallbladder carcinoma than it is with ordinary chronic cholecystitis/cholelithiasis.

XGC arising in non-neoplastic gallbladder disease has been ascribed to bile entering the gallbladder wall, either via ulcers of the surface mucosa or following rupture of Rokitansky-Aschoff sinuses. This contention is supported by the frequent finding of bile and of mucus in the lesion, and by the occasional finding of a focus of XGC with a disrupted sinus at its centre. . $^{1,3,4}$ Gallbladder carcinoma might provide a route for bile to enter the stroma more readily than in chronic cholecystitis or cholelithiasis because of the greater degree of tissue destruction associated with it, providing a simple explanation of the findings in this study. Alternatively, both XGC and carcinoma may be independently associated with chronic gallbladder disease of particular intensity and duration.

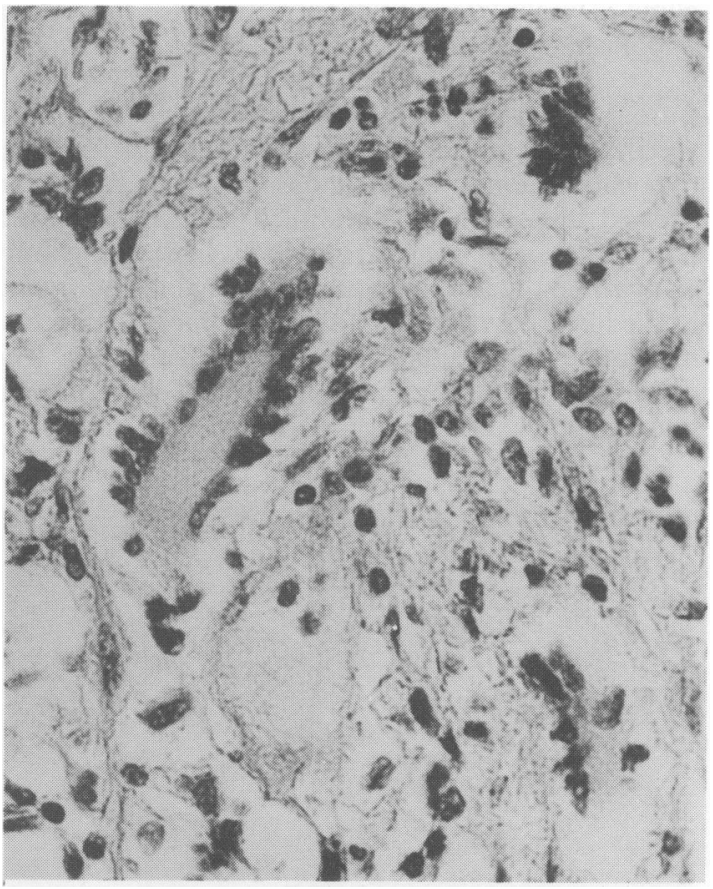

Figure 4 Prominent Touton-type giant cells (Case 1). Haematoxylin and eosin $\times 392$.

Simultaneous XGC and carcinoma of the gallbladder have been described before: of 47 cases of XGC in the referral files of the Armed Forces Institute of Pathology, five also had carcinoma ${ }^{3,4}$ Such referred material is not representative of any defined population, and interesting or unusual cases are likely to be over-represented within it because they catch the imagination of the referring pathologists. Of greater significance is the observation that the suggested diagnosis was sarcoma in two of the non-neoplastic cases ${ }^{4}$ and carcinoma in one. ${ }^{3}$ This ability to simulate neoplasm extends beyond histological appearances: XGC distorts the outline of the gallbladder, forms adhesions with adjacent tissues, and participates in fistula formation. ${ }^{\prime}$ This may cause misdiagnosis as gallbladder carcinoma on pre-operative evaluation and at laparotomy; in particular, XGC may look like a malignant neoplasm on ultrasonography ${ }^{12,13}$ and on computerized axial tomography. ${ }^{14}$

Where XGC and carcinoma coexist, the potential for error increases: the presence of xanthogranulomatous cholecystitis might lead to carcinoma being overlooked or, alternatively, for the stage of the malignant spread to be overestimated. This did not occur in the cases described here, but previous errors in the inter- 
pretation of $\mathrm{XGC}^{3,4}$ emphasize the potential dangers. Apart from cases with minimal mural invasion, gallbladder carcinoma has an appalling prognosis: ${ }^{15}$ it would be a pity to underestimate a patient's chances of survival because of XGC, or to miss the diagnosis of carcinoma altogether.

\section{References}

1. Roberts, K.M. \& Parsons, M.A. Xanthogranulomatous cholecystitis: a clinicopathological study of 13 cases. $J$ Clin Pathol 1987, 40: 412-417.

2. Benbow, E.W. \& Taylor, P.M. Simultaneous xanthogranulomatous cholecystitis and primary adenocarcinoma of gallbladder. Histopathology 1988, 12: 672-675.

3. Goodman, Z.D. \& Ishak, K.G. Xanthogranulomatous cholecystitis. Am J Surg Pathol 1981, 5: 653-659.

4. Christensen, A.H. \& Ishak, K.G. Benign tumors and pseudotumors of the gallbladder. Report of 180 cases. Arch Pathol 1970, 90: 423-432.

5. McCoy, J.J., Vila, R., Petrossian, G., McCall, R.A. \& Reddy, K.S. Xanthogranulomatous cholecystitis. $J$ SC Med Assoc 1976, 72: 78-79.

6. Amazon, K. \& Rywlin, A.M. Ceroid granulomas of the gallbladder. Am J Clin Pathol 1980, 73: 123-127.

7. Reyes, C.V., Jablokow, V.R. \& Reid, R. Xanthogranulomatous cholecystitis: report of seven cases. Am Surg 1981, 47: 322-325.

8. Fligiel, S. \& Lewin, K.J. Xanthogranulomatous cholecystitis. Case report and review of the literature. Arch Pathol Lab Med 1982, 106: 302-304.

9. Mehrotra, M.L. \& Bhatnagar, B.N.S. Biliary granulomatous cholecystitis. J Indian Med Assoc 1982, 79: $144-145$.

\section{Acknowledgements}

I wish to thank Dr Paul Taylor for Figure 1. I am grateful to the staff of the Surgical Histology Department at Manchester Royal Infirmary for their help, and to Margaret Banton for printing the photomicrographs.

10. Takahashi, K., Oka, K., Hakozaki, H. \& Kojima, M. Ceroid-like histiocytic lesion of gall-bladder. A previously undescribed lesion. Acta Pathol Jpn 1976, 26: 25-46.

11. Roberts, K.M. \& Parsons, M.A. Simultaneous xanthogranulomatous cholecystitis and primary adenocarcinoma of the gallbladder (letter). Histopathology 1988, 13: 708.

12. Bluth, E.I., Katz, M.M., Merritt, C.R.B., Sullivan, M.A. \& Mitchell, W.T. Echographic findings in xanthogranulomatous cholecystitis. J Clin Ultrasound 1979, 7: 213-214

13. Gockel, H.P. Xanthogranulomatöse cholezystitis. Fortschr Röntgenstr 1984, 140: 223-224.

14. Düber, C., Störkel, S., Wagner, P.-K. \& Müller, J. Xanthogranulomatous cholecystitis mimicking carcinoma of the gallbladder: CT findings. J Comput Assist Tomogr 1984, 8: 1195-1198.

15. Bergdahl, L. Gallbladder carcinoma first diagnosed at microscopic examination of gallbladders removed for presumed benign disease. Ann Surg 1980, 191: 19-22. 